\title{
Central Nervous System Intravascular Large B-Cell Lymphoma
}

National Cancer Institute

\section{Source}

National Cancer Institute. Central Nervous System Intravascular Large B-Cell Lymphoma. NCl Thesaurus. Code C129602.

A rare extranodal B-cell non-Hodgkin lymphoma that affects the central nervous system. It is characterized by the presence of lymphoma cells exclusively in the lumina of small vessels, particularly capillaries. 